\title{
Triaxial Compressive Strain in Bilayer Graphene Enabled by Nitride Stressor Layer
}

Solomon Mikael ${ }^{1, \dagger}$, Jung-Hun Seo, ${ }^{1, \mathrm{a}), \uparrow}$, Dong-Wook Park ${ }^{1, \dagger}$, Munho Kim ${ }^{1}$, Hongyi Mi ${ }^{1}$, Alireza Javadi $^{2}$, Shaoqin Gong ${ }^{2}$, Zhenqiang Ma ${ }^{1, *}$

${ }^{1}$ Department of Electrical and Computer Engineering, University of Wisconsin-Madison, Madison, WI 53706, USA

${ }^{2}$ Department of Biomedical Engineering, Wisconsin Institute for Discovery, and Materials Science Program, University of Wisconsin-Madison, Madison, WI 53706, USA

${ }^{a}$ Current address: Department of Materials Design and Innovation, University at Buffalo, The State University of New York, Buffalo, NY 14260, USA

${ }^{\dagger}$ These authors contributed equally to this work.

"Correspondence should be addressed to Zhenqiang Ma (ㅎazq@engr.wisc.edu $)$

Keywords: Triaxial compressive strain; Strained Graphene; Nitride Stressor Layer; Raman Spectroscopy

\begin{abstract}
A technique that can be used to viably create triaxially strained bilayer graphene on any desirable location by simple patterning was developed. Unlike the conventional graphene strain engineering methods, the photolithographically defined spoke patterns and compressive strained $\mathrm{Si}_{3} \mathrm{~N}_{4}$ layer deposited by plasma-enhanced chemical vapor deposition (PECVD) system enable the creation of locally confined triaxial strained bilayer graphene at the desire location by forming a unique tristar shaped wrinkle. The tristar shaped wrinkle was investigated with high resolution micro-Raman spectroscopy and atomic force microscopy (AFM) analyses, and confirmed that $0.38 \%$ of maximum triaxial compressive strain was created. Mechanical simulation was used to verify the strain distribution and confirm the strain value which was calculated from the Raman spectroscopy and AFM profile. The technique presented here not only provides a practical route to the creation of strained graphene at desired locations but also offers the potential of the creation of multiaxial strain
\end{abstract}


in the graphene for various types of graphene-based electronic and optoelectronic devices.

\section{Introduction}

Strain engineering of bilayer graphene has been researched as the one of the key approaches to utilize graphene as a useful material for electronics and optoelectronic applications [1-5]. The bandgap opening of the strained graphene has been theoretically calculated over $100 \mathrm{meV}$ and experimentally investigated [6-9]. At an early stage, the strain in graphene was realized by suspending it on 1-dimensional or 2-dimensional textured surfaces, such as ripples or staircase pattern [10-13]. Recently, advanced approaches have been proposed experimentally and theoretically to create the uniaxial, biaxial or triaxial strain in the 2-D materials such as graphene, including bending or deforming the substrate, or employing thermal expansion of graphene [14-20]. Although these approaches have been successfully demonstrated the uniaxial, biaxial or triaxial strain in the graphene and their bandgap opening, the strain distributions were uncontrolled and difficult to predict where they will form. Moreover, these methods not only require very complicated fabrication processes and/or specific conditions in order to generate, but often fail to provide permanent strain in graphene. From the practical point of view, therefore, it is necessary to develop a new method that can create and define the strain at the desired location in order to fully utilize improved characteristics of bilayer graphene.

In this paper, we report a simple and viable method to generate triaxial strain in bilayer graphene at the desire location by using a conventional patterning and deposition technique. The location of the strain in the bilayer graphene was easily controlled by a spoke shaped pattern. The use of $\mathrm{Si}_{3} \mathrm{~N}_{4}$ stressor layer allows us to manipulate the desired intensity of the strain in the bilayer graphene. Additionally, as proposed in a Figure 2, the various types of strains, namely from biaxial to multiaxial strain, can be simply realized by changing the shape of the spoke pattern. As an example demonstration in this paper, a pattern with three spokes was employed and successfully created the tristar shaped wrinkle at the center of the spokes. The unique tristar shape wrinkle formed at the center of spokes by compressivly strained samples enables us to realize triaxial strain in bilayer graphene for the first time. The triaxial strain created using this technique influences the optical phonon properties of bilayer graphene and such changes were characterized by using high resolution micro-Raman spectroscopy and atomic force microscopy (AFM). Mechanical simulation was used to reveal 
the strain distribution and the simulation result agrees well with the strain values calculated from the Raman spectroscopy and AFM surface profile analyses.

\section{Experiment}

\subsection{Graphene growth.}

The graphene was grown on a copper foil in a home built low pressure chemical vapor deposition (LPCVD) system. The copper was placed in the quartz tube with only hydrogen flowing. The copper foil samples were annealed at $1050{ }^{\circ} \mathrm{C}$ for 20 minutes in a hydrogen ambient. Following the annealing, the growth was initiated by adding methane to the gas mixture for an additional 20 minutes with a ratio of $1: 1$. During the graphene growth period, a low pressure $(<1$ Torr) was maintained for the first half of the processing time and then, a high pressure (>1 Torr) was applied for the remaining time. The growth conditions outlined above produced high quality graphene with dense bilayer graphene regions. The graphene was transferred onto $\mathrm{SiO}_{2}$ using PMMA as a transfer membrane. PMMA was spin-coated onto one side of the $\mathrm{Cu}$ foil with graphene and baked for 2 minutes at $150{ }^{\circ} \mathrm{C}$. The graphene on the backside of the $\mathrm{Cu}$ foil was removed with $\mathrm{O}_{2}$ plasma etching. The stack consisting of $\mathrm{Cu}$ foil/graphene/PMMA was then placed in $\mathrm{FeCl}_{3}$ to etch away the $\mathrm{Cu}$. The remaining graphene/PMMA film was then rinsed and placed onto a Si wafer.

\subsection{Deposition of the Silicon nitride stressor layer.}

A mixture of $2 \%$ silane $\left(\mathrm{SiH}_{4}\right)$ in $\mathrm{N}_{2}, 5 \%$ ammonia $\left(\mathrm{NH}_{3}\right)$ in $\mathrm{N}_{2}$, and Nitrous oxide $\left(\mathrm{N}_{2} \mathrm{O}\right)$ react to form silicon nitride with different amounts of stress. The detailed $\mathrm{Si}_{3} \mathrm{~N}_{4}$ deposition parameters are given in Table 1. Each sample was post-annealed for 5 minutes in an $\mathrm{N}_{2}$ ambient followed by 3 minutes in high vacuum. In both cases, silicon nitride films with a thickness of $25 \mathrm{~nm}$ were deposited and measured using an optical reflectometer (Filmetrics F20). Changes in film stress were measured by using a stress measurement system (Tencor FleXus FLX-2320). The stresses induced by the silicon nitride layer with and without the bilayer graphene were determined by measuring the change in the curvature of the Si sample substrate, and relating stress to curvature by the Stoney approximation equation (1):

$$
\sigma=\frac{E}{1-v} \cdot \frac{t_{s}^{2}}{6 \cdot t_{f}} \cdot\left(\frac{1}{R_{1}}-\frac{1}{R_{2}}\right)
$$


where $E$ is the Young's modulus, $v$ is the Poisson ratio, $R_{l}$ is the radius of the initial curvature of the layered structure (i.e. the graphene film covered with a $20 \mathrm{~nm} \mathrm{SiO} 2$ protection layer on the $\mathrm{SiO}_{2} / \mathrm{Si}$ wafer), $R_{2}$ is the curvature after the deposition of the $\mathrm{Si}_{3} \mathrm{~N}_{4}$ stressor film, $t_{\mathrm{s}}$ is the thickness of the silicon substrate, and $t_{f}$ is the thickness of the thin film producing the stress (i.e. the thickness of the $\mathrm{Si}_{3} \mathrm{~N}_{4}$ layer in this experiment). Equation 1 is only valid when $t_{s}$ is larger than $t_{f}$. The $\mathrm{Si}_{3} \mathrm{~N}_{4}$ films generate a maximum compressive stress of $840 \mathrm{MPa}$ on the graphene for different deposition conditions. $\mathrm{Si}_{3} \mathrm{~N}_{4}$ film PECVD parameters and the stress value were measured from the layered samples consist of $25 \mathrm{~nm} \mathrm{Si}{ }_{3} \mathrm{~N}_{4} / 20 \mathrm{~nm} \mathrm{SiO} / 200 \mathrm{~nm}$ $\mathrm{SiO}_{2} / \mathrm{Si}$ substrate.

Table 1. $\mathrm{Si}_{3} \mathrm{~N}_{4}$ film PECVD parameters and the measured stress on the layered samples consisting of $25 \mathrm{~nm} \mathrm{Si}_{3} \mathrm{~N}_{4} / 20 \mathrm{~nm} \mathrm{SiO}_{2} / 100 \mathrm{~nm} \mathrm{SiO}_{2} /$ Si substrate.

\begin{tabular}{c|c|c|c|c|c|c|c} 
& $\begin{array}{c}2 \% \mathrm{SiH}_{4} \\
\text { flow } \\
(\mathrm{sccm})\end{array}$ & $\begin{array}{c}\mathrm{NH}_{3} \text { flow } \\
(\mathrm{sccm})\end{array}$ & $\begin{array}{c}\mathrm{N}_{2} \mathrm{O} \text { flow } \\
(\mathrm{sccm})\end{array}$ & $\begin{array}{c}\text { Pressure } \\
(\mathrm{mTorr})\end{array}$ & $\begin{array}{c}\mathrm{RF} \text { Power } \\
(\mathrm{W})\end{array}$ & $\begin{array}{c}\text { Temp. } \\
\left(\mathrm{C}^{\circ}\right)\end{array}$ & $\begin{array}{c}\text { Measured stress } \\
(\mathrm{MPa})\end{array}$ \\
\hline $\begin{array}{c}\text { High } \\
\text { Compressive stress }\end{array}$ & 250 & 50 & 10 & 950 & 26 & 350 & 840 \\
\hline $\begin{array}{c}\text { Low } \\
\text { Compressive stress }\end{array}$ & 175 & 125 & 10 & 950 & 46 & 350 & 530 \\
\hline Reference un-stress & 50 & 20 & 810 & 950 & 46 & 350 & 15
\end{tabular}

\subsection{Fabrication of triaxial strained graphene}

The schematic illustration of the device fabrication is shown in Figure 1(a). The process began with cleaning the $100 \mathrm{~nm} \mathrm{SiO}_{2} / \mathrm{Si}$ substrate, followed by the layer transfer of bilayer graphene. The growth and transfer processes of chemical vapor deposition (CVD) grown bilayer graphene can be found elsewhere [21]. The bilayer graphene was defined to hexagonal shaped with $20 \mathrm{~nm}$ thick $\mathrm{SiO}_{2}$ etching protection layer by photolithography. The hexagonally patterned bilayer graphene was then firmly tied by using titanium clamps in order to prevent bilayer graphene layer from sliding after the deposition of a stressor $\mathrm{Si}_{3} \mathrm{~N}_{4}$ layer. The $25 \mathrm{~nm}$ thick $\mathrm{Si}_{3} \mathrm{~N}_{4}$ stressor layer was deposited on the entire surface including the patterned bilayer graphene by using conventional plasma enhanced chemical vapor deposition (PECVD) system. A mixture of $2 \%$ silane $\left(\mathrm{SiH}_{4}\right)$ in $\mathrm{N}_{2}, 5 \%$ ammonia $\left(\mathrm{NH}_{3}\right)$ in $\mathrm{N}_{2}$, and Nitrous oxide $\left(\mathrm{N}_{2} \mathrm{O}\right)$ react to form silicon nitride with different amounts of stress. Figure 1(f) illustrates how wrinkles are created by compressive strain and its effect on the graphene 
layer. The bottom graphene is strongly bonded to the substrate. The graphene samples were transferred on to the $\mathrm{SiO}_{2} / \mathrm{Si}$ substrate, and Van der Waals forces [22-23] and hydrogen bonding [24], which is much stronger than the interlayer bonding strength between the bottom layer and the top layer of graphene, hold this layer firmly in place. Due to the loosely bonded interlayer, only the top graphene layer can be shrunk by " $\Delta \ell$ " by compressive strains. In other words, the compressive $\mathrm{Si}_{3} \mathrm{~N}_{4}$ stressor layer applied compressive stress to both the top and bottom graphene layers simultaneously. However, due to the weak interlayer bonding force between the top and the bottom graphene layers, the top graphene layer experienced less compression. The difference in force is mostly concentrated near the central region (i.e., the force neutral region) and caused delamination of the $\mathrm{Si}_{3} \mathrm{~N}_{4}$ stressor layer and the top graphene layer to form wrinkles. In this way, as illustrated in Figure 2, the creation of multiaxial strains (for instance, biaxial strain, triaxial strain, quadriaxial strain and even quintaxial axial strain) are possible. Although this paper only presents compressively strained bilayer graphene, our preliminary study showed that it is possible to create a tensile-strained graphene layer using the same approach. In other words, the type (compressive strain and tensile strain) and the amount of stress generated in the bilayer graphene by the $\mathrm{Si}_{3} \mathrm{~N}_{4}$ film can be easily manipulated by changing the deposition conditions of the $\mathrm{Si}_{3} \mathrm{~N}_{4}$ film. We believe that the method proposed in this paper can be globally applied from monolayer to multilayer graphene. However, as described in the mechanism of the formation of wrinkles, the strain will be more effective for bilayer and multilayered graphene, although the strain can be applied to monolayer graphene because the $\mathrm{Si}_{3} \mathrm{~N}_{4}$ stressor layer can mechanically deform the substrate."

In this experiment, two types of compressive stresses were used; namely the $840 \mathrm{MPa}$ and the $530 \mathrm{MPa}$ for the high stress and low stress $\mathrm{Si}_{3} \mathrm{~N}_{4}$ film, respectively. It is important to note that these measurements were carried out using samples consisting of $25 \mathrm{~nm} \mathrm{Si}_{3} \mathrm{~N}_{4} / 20$ $\mathrm{nm} \mathrm{SiO}_{2} / 100 \mathrm{~nm} \mathrm{SiO} / 2 \mathrm{Si}$ substrate because wrinkles formed on the films in the presence of the graphene layers, as elaborated in the next paragraph, which adversely affected the accuracy of these measurements. The illustration shown in Figure 1(e) presents the mechanism of the generation of strain showing the tristar shape wrinkle forms at the central intersection of three strips. As a reference, the sample with the very low $\mathrm{Si}_{3} \mathrm{~N}_{4}$ compressive stress of $15 \mathrm{MPa}$ was prepared and denoted as "un-strain". We believe that the types and amount of stress generated in the films consisting of the $25 \mathrm{~nm} \mathrm{Si}{ }_{3} \mathrm{~N}_{4} / 20 \mathrm{~nm} \mathrm{SiO}$ /bilayer graphene/100 $\mathrm{nm} \mathrm{SiO} 2 / \mathrm{Si}$ substrate would be very similar to these data measured without the 
bilayer graphene layer, because the graphene layer is not only extremely thin in comparison with the $\mathrm{Si}_{3} \mathrm{~N}_{4}$ layer, but the Van der Waals force also makes the graphene layer strongly adhere to the silicon substrate due to their intimate contact. Annealing the samples at $350{ }^{\circ} \mathrm{C}$ during the $\mathrm{Si}_{3} \mathrm{~N}_{4}$ deposition process further enhances the adhesion between the substrate and graphene due to additional hydrogen bonding [24].

(a)

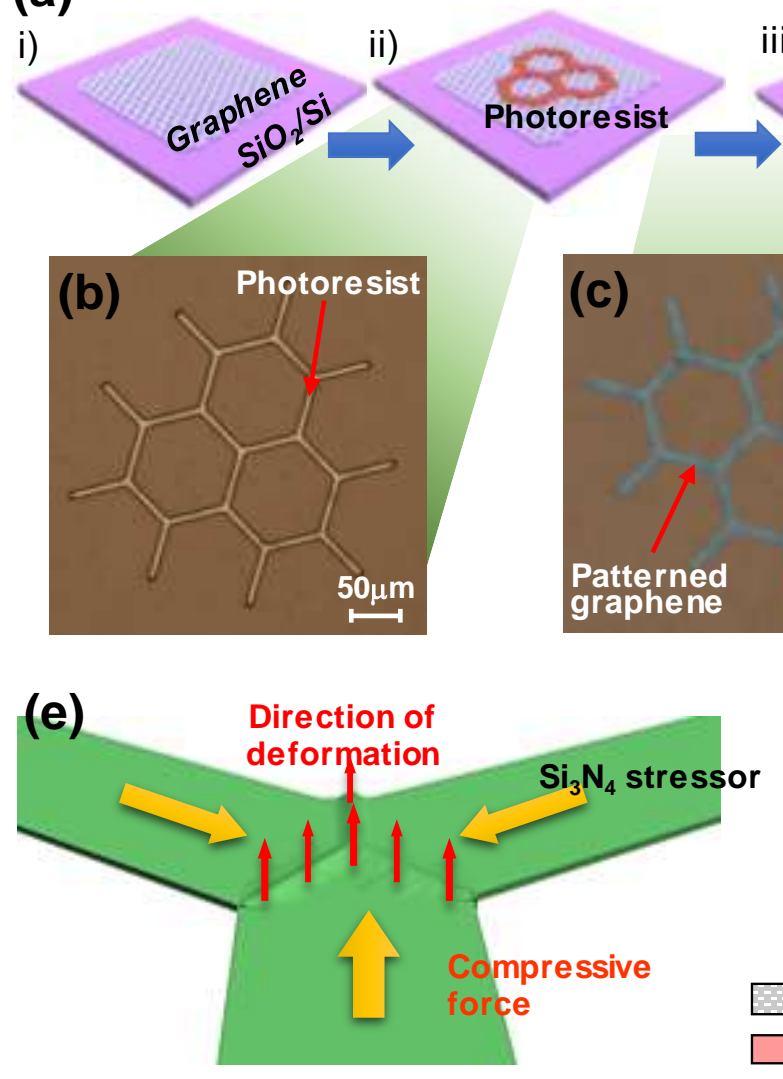

iii)

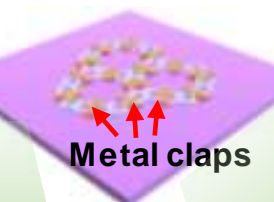

etal claps
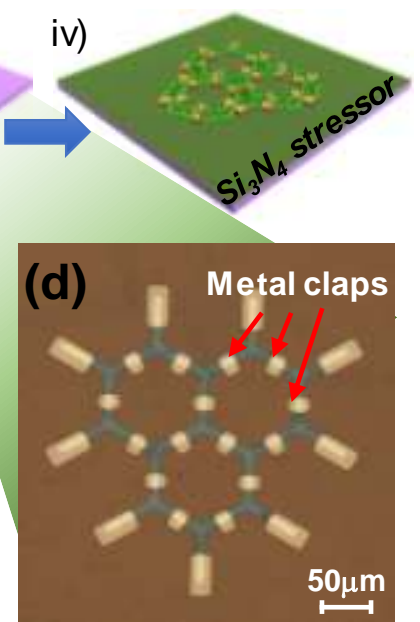

(f)

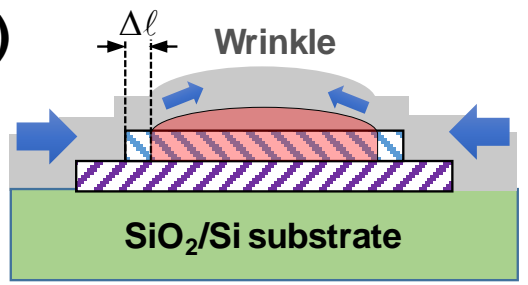

$\mathrm{Si}_{3} \mathrm{~N}_{4}$ layer $\square$ Unstrained top graphene Strained top graphene $\square$ Bottom graphene
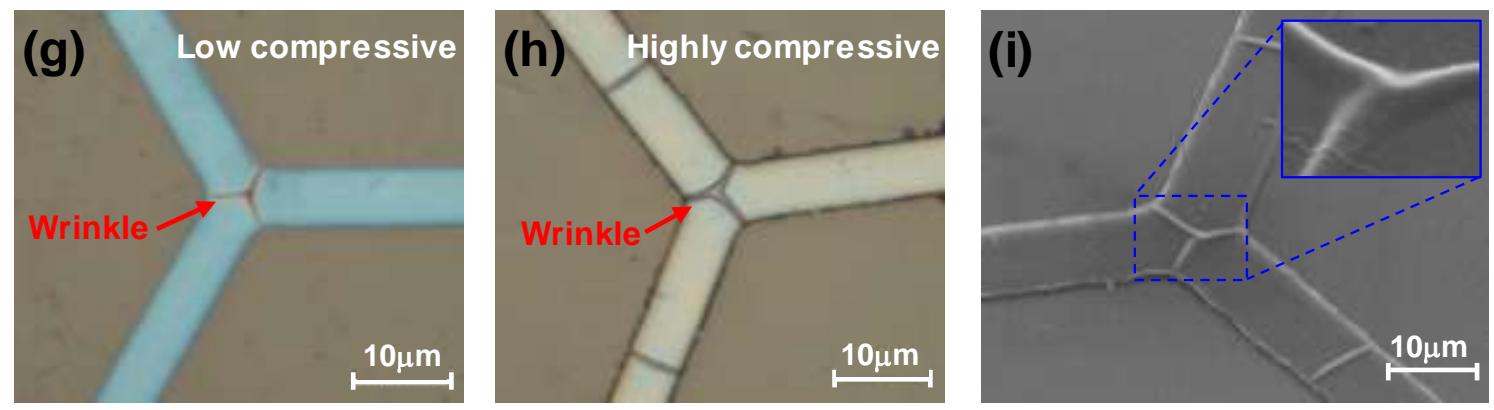

Figure 1. (a) schematic illustrations and images of the fabrication process for creating triaxial compressive strain in bilayer graphene. (i) Preparation of the CVD grown bilayer graphene. (ii) A hexagonal shape patterning on a bilayer graphene. (iii) Deposition of $\mathrm{Cr}$ claps to fix the patterned graphene layer. (iv) Deposition of $\mathrm{Si}_{3} \mathrm{~N}_{4}$ stressor layer on entire surface to apply a strain. (b)-(d) Microscopic images, corresponding to step (ii) - (iii). (e) 
An illustration to show the mechanism of the formation of tristar shape wrinkle. (f) An illustration showing the formation of wrinkles by compressive Si3N4 stressor layer. (g)(h) Microscopic images after the deposition of low and high $\mathrm{Si}_{3} \mathrm{~N}_{4}$ stressor layers. Wrinkles are formed clearly. (i) A tilted SEM image taken at the tristar shaped wrinkle.

Figure 1(b)-(d) show the microscopic images taken during the fabrication process (Figure 1(c), (e) correspond to the step ii and iii in Figure 1(a), various images of wrinkled graphene can be found in Figure 2). Figure 1(g) and (h) were taken after the deposition of the low (530 MPa) and high (840 MPa) compressive stressed $\mathrm{Si}_{3} \mathrm{~N}_{4}$ layers, respectively. Figure 1(i) shows the angled scanning electron microscope (SEM) image which indicates the tristar shape wrinkles with a height of $\sim 70 \mathrm{~nm}$ (See the detailed measurement and calculation in Figure 5 (c)). Notably, the tristar shape wrinkle was formed at the central intersection region where the compressive stress from the three strips were concentrated.

(a)

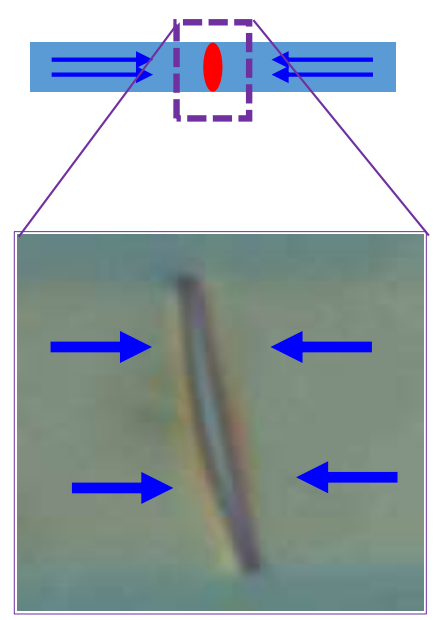

(b)

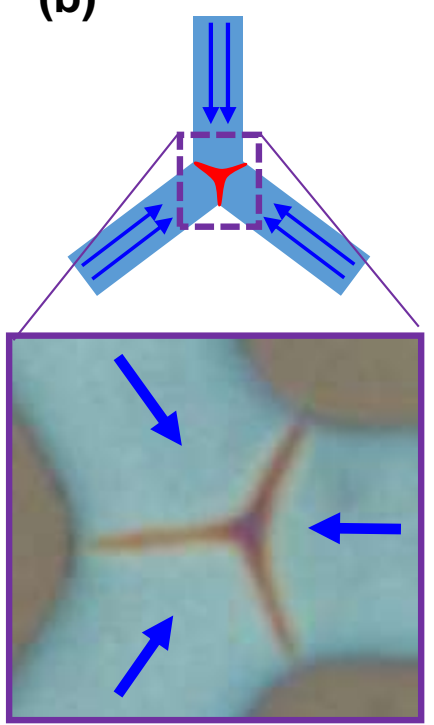

(c)

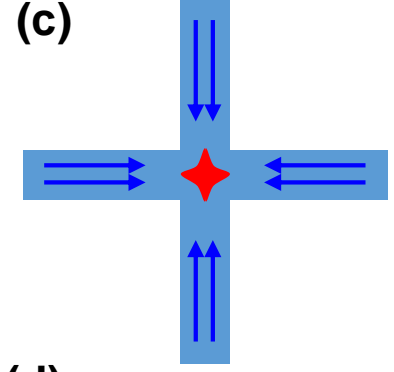

(d)

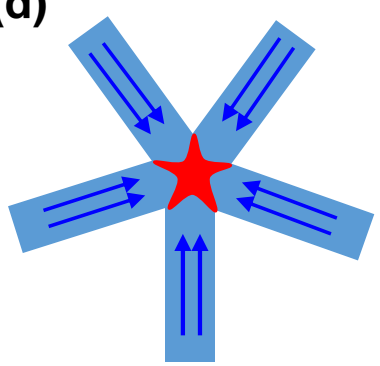

Figure 2. Graphical illustration of the method of creating multiaxial strain by patterning various number of spokes to generate (a) biaxial strain, (b) triaxial strain, (c) quadriaxial strain and (d) quintaxial axial strain, respectively, as examples. Microscopic images for Figure 2 (a) and (b) show the actual shapes of wrinkles formed by biaxial and triaxial strain, respectively. 


\section{Results and discussions}

The triaxial strain of the patterned bilayer graphene samples was investigated by using a Horiba micro-Raman spectroscopy (spectrometer resolution of $0.045 \mathrm{~cm}^{-1}$ ) with a $50 \times$ objective lens (a spot size of about $1 \mu \mathrm{m}$ ) and $18.5 \mathrm{~mW}$ of He-Ne $(532 \mathrm{~nm}$ ). Raman spectroscopy has been widely used to investigate the phonon vibration properties of graphene. The influence on phonon vibration of graphene by mechanical strain can be correlated with the change in Raman characteristic peaks of graphene. Therefore, the actual strain applied to the graphene layer can be accurately calculated by the Gruneisen parameter and the measured Raman shifts $[10,25]$. The amount of compressive strain that was applied to the bilayer graphene at the center of tristar wrinkle was extracted by the following equation $[10,25]$ :

$$
\gamma=-\frac{\Delta \omega}{\omega_{0}} \cdot \frac{1}{\delta \varepsilon_{h}}
$$

where $\omega_{0}$ is the Raman band frequency without strain, $\delta \omega$ is the Raman band shift, $\gamma$ is Grünei sen parameter for the corresponding band, and $\varepsilon_{h}$ is the hydrostatic strain in the graphene film. For triaxial strain, $\varepsilon_{h}$ can be expressed as $\varepsilon_{h}=\varepsilon_{1}+\varepsilon_{2}+\varepsilon_{3}$, i.e., the sum of the three directional strain components of the strain, and these three components have the same value $\left(\varepsilon_{1}=\varepsilon_{2}=\varepsilon_{3}\right)$. According to Equation (2), based on the previously reported Grüneisen parameter [25] and the blue-shift in the $\mathrm{G}$ band peak, the calculated triaxial compressive strain was calculated to be $0.38 \%$. This averaged triaxial strain value extracted from Raman shift agrees well with the value of $0.4 \%$ derived from the angled SEM and AFM image (see Figure 5(c)).

(a)

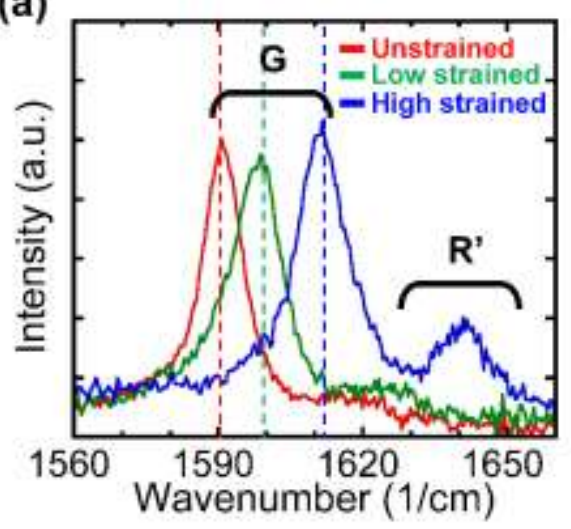

(b)

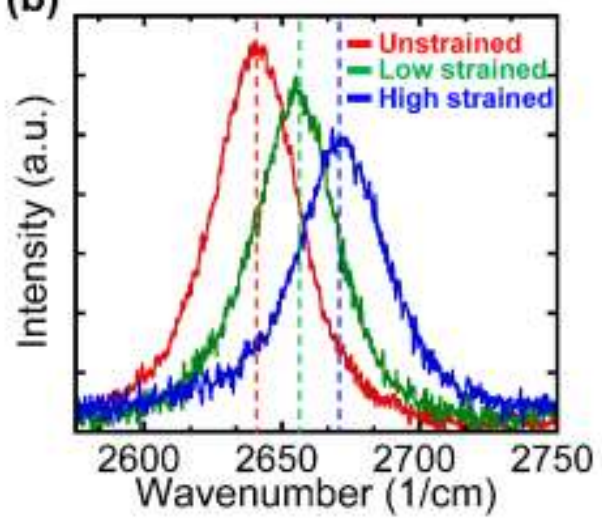


Figure 3. Raman shifts of (a) $G$ band and (b) 2D band on the tristar shape wrinkled graphene induced by the $\mathrm{Si}_{3} \mathrm{~N}_{4}$ compressive stressor with different degree of strains (Red: un-strained, Green: low strained, Blue: high strained).

Raman spectra taken from the sample with un-strained, low strained, and high strained $\mathrm{Si}_{3} \mathrm{~N}_{4}$ layer were shown in Figure 3. Both $\mathrm{G}$ band and 2D band peaks clearly show the blueshifting as the compressive strain value increases. $G$ band peaks started to shift when the low strained $\mathrm{Si}_{3} \mathrm{~N}_{4}$ stressor layer was applied, and $\sim 31 \mathrm{~cm}^{-1}$ of shifting was observed when the high strained $\mathrm{Si}_{3} \mathrm{~N}_{4}$ stressor layer was applied. In addition to the $\mathrm{G}$ peak, the $\mathrm{R}^{\prime}$ peak was also observed at the higher wavenumber to the $G$ peak. The $R^{`}$ peak is the sub-peak as a result of the twisting between the top and the bottom graphene layer, and its' intensity increases as the amount of strain applied to the graphene increases. In the Raman spectrum of graphene, the $G$ band is related to the doubly degenerated $\mathrm{E}_{2 \mathrm{~g}}$ at the center of the Brillouin zone, while the $2 \mathrm{D}$ band is related to the momentum conservation of the scattering of two phonons with opposite waver vectors $[25,26]$. Thus, strain can influence the phonon variation in the crystal structure of graphene [27]. Specifically, the change in phonon vibration at the center-zone and the change in the double-resonance condition by the triaxial strain causes the $\mathrm{G}$ band and the $2 \mathrm{D}$ band to shift.

(a)

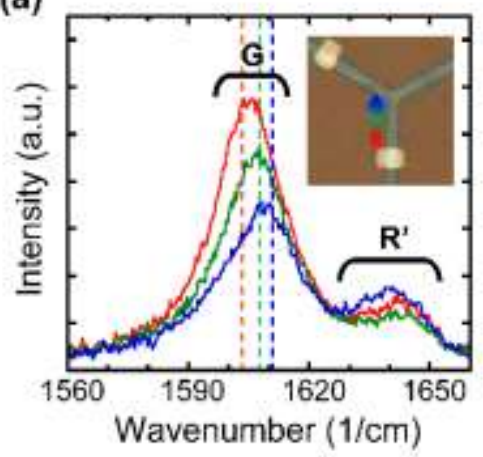

(b)

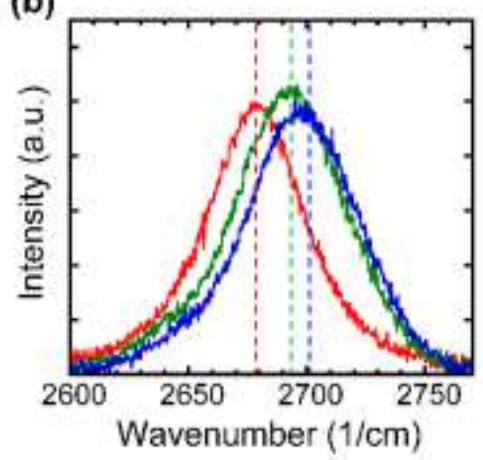

(c)

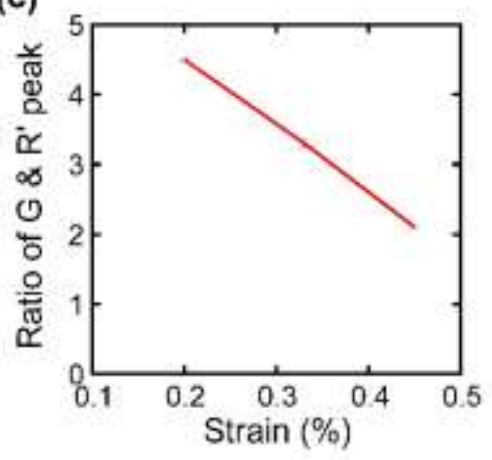

Figure 4. Raman shifts of (a) G band and (b) 2D band measured by line scanning from the tristar wrinkle at the highly strained sample, showing $\mathrm{R}^{\prime}$ band became more distinctive as the spot moved to the central intersection of three strips. Inset shows the actual scanning direction. The colors in the arrow match with the color of each plots. (c) Ratio of G peak and R' peak as a function of strain from Figure 3(a). 
As previously reported, the $G$ band splits into $G$ and $G$ ' bands and blue-shifts under compressive strain. For uniaxial strain, the $\mathrm{G}$ band splits into two peaks and the $\mathrm{G}^{\prime}$ band can broaden [28-29]. For biaxial or triaxial strain, in contrast, the widths of the $G$ band is unaffected by strain, but it is caused by the interaction of electrons, LO phonons, and interior folded phonons at the intravalley [30]. Lu et al. revealed that the $\mathrm{R}^{\prime}$ peak is attributed to the twisting between two graphene layers with a twist angle of $3-8^{\circ}$ [31]. This $\mathrm{R}^{\prime}$ peak is caused by a double intravalley resonance process and is only activated in the visible range. Interestingly, we found that the ratio between $G$ peak and $R$ ' peak decreased, as the measurement point approaches to the tristar wrinkled center or, in other words, as triaxial strain increases. Figure 4 (c) clearly shows the linear correlation between the ratio of $G$ and $\mathrm{R}^{\prime}$ peaks and the actual strain applied to the graphene layer as a result of the twisted angle between two graphene layers. The addition of the straining film has not only shifted the $G$ peak of the bilayer graphene but has also induced some twisting of $\sim 7^{\circ}$, this clearly indicates that the graphene is under a great deal of compressive stress.

To further investigate the differences of triaxial strain on various spots near the center region, the line scanning of Raman spectroscopy on the graphene was performed. The microscopic image of the scanning direction near the center region is shown in an inset of Figure 4(a). Raman spectra shown in Figure 4(a) and (b) demonstrated that the graphene film displayed significant blue-shifts in $\mathrm{G}$ band $\left(2.4 \mathrm{~cm}^{-1} / \%\right)$ and $2 \mathrm{D}$ band $\left(4.4 \mathrm{~cm}^{-1} / \%\right)$ with clear $\mathrm{R}^{\prime}$ band formation, which is undoubtedly attributed to the tri-directional compressions to the center. The blue-shifts of $\mathrm{G}$ and 2D bands were measured from the other tristar wrinkled centers of the graphene and showed consistent shifts.

As shown in Figure 5(a), the morphology of the wrinkled graphene was carefully measured by a non-contact mode AFM over a $20 \times 20 \mu \mathrm{m}^{2}$ area. The center part of the strained graphene film clearly shows tristar shape triaxial strained graphene with about $70 \mathrm{~nm}$ in height. The small wave-like wrinkles on each arm that is shown in the inserted 2D graphene surface profile in Figure 5(a) suggested that the highest strain intensity was concentrated at the center of the wrinkle, but small strains also existed on the arms where the $\mathrm{Si}_{3} \mathrm{~N}_{4}$ stressor layer was deposited. In order to analyze and evaluate the tristar shaped str ain distribution at the center region, a strain simulation was carried out by COMSOL Multiphysics with a solid stress strain model. As shown in Figure 5(b), we incorporated the actual dimensions, structure, and material parameters which were analyzed by AFM into 
the simulation model. For graphene, we employed Young's modulus of $1 \mathrm{TPa}$, and Poisson's ratio of 0.3 [32-33]. We also used $0.14 \mathrm{GPa}$ of the interlayer shear strength of between the bottom layer and the top layer graphene to replicate the bilayer graphene [34]. It should be noted that all parameters were taken from the experimental values. Once we established the structure, various compressive strains were applied to the graphene stack to reproduce the actual shape of tristar wrinkled graphene as measured in Figure 5(c). As shown in Figure 5(b), the simulated results correspond well with the calculated results based on the values from the Raman spectroscopy and AFM surface profile analyses.

(a)

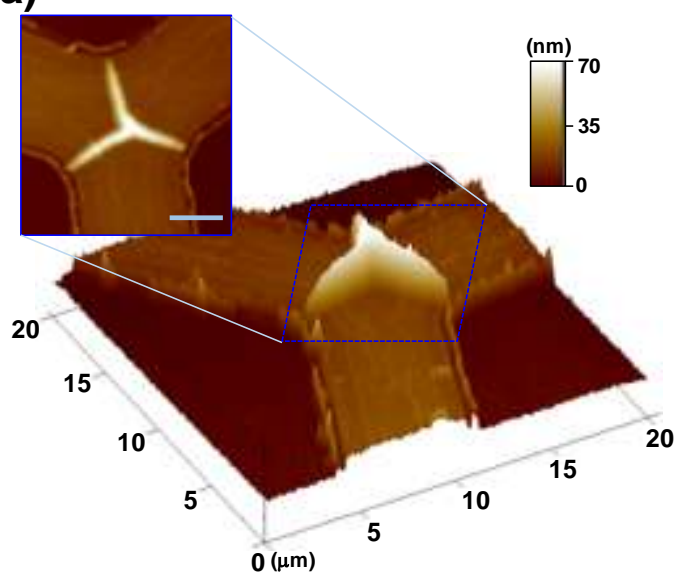

(b)

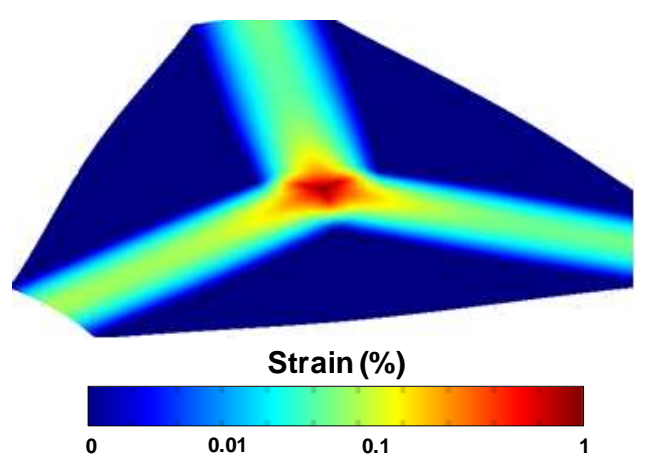

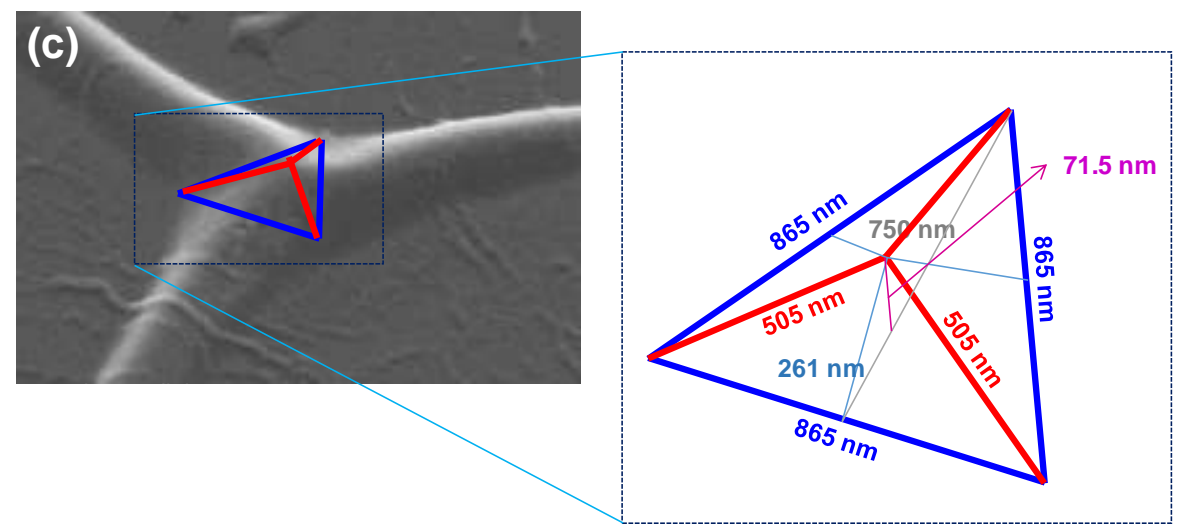

Height of wrinkle $: 71.5 \mathrm{~nm}$

Figure 5. (a) An AFM image to show the three dimensional surface profile of tristar shape wrinkled bilayer graphene after the deposition of a $\mathrm{Si}_{3} \mathrm{~N}_{4}$ compressive stressor layer. Inset show the top view of the scanned region. (b) Simulated triaxial compressive strained graphene with high compressive stressed $\mathrm{Si}_{3} \mathrm{~N}_{4}$ layers by COMSOL 
Multiphysics. (c) A measurement of the dimension of tristar wrinkle (Left) by SEM image and (Right) calculation of its' height.

\section{Conclusion}

In summary, we have developed the technique that can viably create the triaxial strained bilayer graphene by using tristar shaped patterning. Unlike the conventional strain engineering method to graphene, our technique allows us to define the strain at the desire location by simple patterning, which, in other words, gives more flexibility and freedom to apply strain at the local regions. Therefore, the technique presented here could be readily applied a triaxial strain not only for various types of graphene-based electronic and optoelectronic devices but also with other two dimensional materials.

\section{Acknowledgements}

This work was supported by ONR under Grant No. N00014-09-1-0803. The program manager at ONR is Dr. Chagaan Baatar. S. Mikael was partly supported by GERS Fellowship and Winslow Sargeant Fellowship. 


\section{References}

[1] K. S. Kim et al. Large-scale pattern growth of graphene films for stretchable transparent electrodes. Nature 457, (2009) 706-710.

[2] G. Jo et al. The application of graphene as electrodes in electrical and optical devices. Nanotechnology, 23 (2012) 112001.

[3] Y. Zhu, et al. Graphene and graphene oxide: synthesis, properties, and applications. Advanced Materials, 22 (2010) 3906-3924.

[4] F. Bonaccorso, Z. Sun, T. Hasan, A. C. Ferrari, Graphene photonics and optoelectronics. Nature Photon. 4, (2010) 611-622.

[5] T. Mueller, F. N. A. Xia, P. Avouris, Graphene photodetectors for high-speed optical communications. Nature Photon. 4, (2010) 297-301.

[6] Z. H. Ni et al. Uniaxial strain on graphene: Raman spectroscopy study and band-gap opening. ACS Nano 2, (2008) 2301-2305; erratum 3, (2009) 483.

[7] S.-M. Choi, S.- H. Jhi, Y.- W. Son, Effects of strain on electronic properties of graphene. Phys. Rev. B 81, (2010) 081407.

[8] T. M. G. Mohiuddin et al. Uniaxial strain in graphene by Raman spectroscopy: G peak splitting, Gruneisen parameters, and sample orientation. Phys. Rev. B 79, (2009) 205433.

[9] S.-M. Choi, S.-H. Jhi, Y-W. Son, Controlling energy gap of bilayer graphene by strain. Nano Lett. 10, (2010) 3486-3489.

[10] C. Metzger et al. Biaxial strain in graphene adhered to shallow depressions. Nano Lett. $10,(2010) 6-10$.

[11] A. Reserbat-Plantey et al. Strain superlattices and macroscale suspension of graphene induced by corrugated substrates. Nano Lett. 14, (2014) 5044-5051.

[12] H. Mi et al. Creating periodic local strain in monolayer graphene with nanopillars patterned by self-assembled block copolymer. Appl. Phys. Lett., 107 (14), (2015) 143107.

[13] J. Bai et al. Graphene nanomesh. Nature Nanotechnol. 5, (2010) 190-194.

[14] W. Pan et al. Biaxial compressive strain engineering in graphene/boron nitride heterostructures. Sci. Rep. 2, (2012) 893.

[15] T. Yu et al. Raman mapping investigation of graphene on transparent flexible substrate: The strain effect J. Phys. Chem. C, 112, (2008) 12602-12605.

[16] H. Lim, J. Jung, R. S. Ruoff, Y. Kim, Structurally driven one-dimensional electron confinement in sub-5-nm graphene nanowrinkles. Nat. Commun., 6, (2015) 8601. 
[17] C. Si, Z. Suna, F. Liu, Strain engineering of graphene: a review. Nanoscale 8, (2016) 3207-3217.

[18] M. Neek-Amal, L. Covaci, K. H. Shakouri, F. M. Peeters, Electronic structure of a hexagonal graphene flake subjected to triaxial stress. Phys. Rev. B, 88, (2013) 115428.

[19] F. Guinea, M. I. Katsnelson, A. K. Geim, Energy gaps and a zero-field quantum Hall effect in graphene by strain engineering. Nature Phys. 6, (2010) 30 - 33.

[20] S. Zhu, J. A. Stroscio, T. Li, Programmable Extreme Pseudomagnetic Fields in Graphene by a Uniaxial Stretch. Phys. Rev. Lett. 115, (2015) 245501.

[21] S. Mikael, J.-H. Seo, A. Javadi, S. Gong, Z. Ma, Wrinkled bilayer graphene with wafer scale mechanical strain. Appl. Phys. Lett. 108, (2016) 183101.

[22] S. P. Koenig, N. G. Boddeti, M. L. Dunn and J. S. Bunch, Ultrastrong adhesion of graphene membranes. Nature Nanotech. 6, (2011) 543-546.

[23] A. B. Kuzmenko, I. Crassee, D. van der Marel, P. Blake and K. S. Novoselov, Determination of the gate-tunable band gap and tight-binding parameters in bilayer graphene using infrared spectroscopy. Phys. Rev. B 80, (2009)165406.

[24] K. Nagashio, T. Yamashita, T. Nishimura, K. Kita, A. Toriumi, Electrical transport properties of graphene on $\mathrm{SiO}_{2}$ with specific surface structures. J. Appl. Phys., 110, (2011) 024513.

[25] T. M. G. Mohiuddin et al. Uniaxial strain in graphene by Raman spectroscopy: G peak splitting, Gruneisen parameters, and sample orientation. Phys. Rev. B 79, (2009) 205433.

[26] F. Ding et al. Stretchable graphene: a close look at fundamental parameters through biaxial straining. Nano Lett. 10, (2010) 3453-3458.

[27] G. Gui, D. Morgan, J. Booske, J. Zhong, Z. Ma, Local strain effect on the band gap engineering of graphene by a first-principles study. Appl. Phys. Lett. 106, (2015) 053113.

[28] O. Frank et al. Raman 2D-band splitting in graphene: theory and experiment. ACS Nano 5, (2011) 2231-2239.

[29] H. Shioya, M. F. Craciun, S. Russo, M. Yamamoto, S. Tarucha, Straining Graphene Using Thin Film Shrinkage Methods. Nano Lett. 14, (2014) 1158-1163.

[30] V. Carozo et al. Raman signature of graphene superlattices. Nano Lett. 11, (2011) 45274534.

[31] C.-C. Lu et al. Twisting bilayer graphene superlattices. ACS Nano 7, (2013) 2587-2594.

[32] C. Lee, X. Wei, J. W. Kysar, J. Hone, Measurement of the elastic properties and intrinsic strength of monolayer graphene. Science 321, (2008) 385-388. 
[33] E. Cadelano, P. L. Palla, S. Giordano, L. Colombo, Nonlinear Elasticity of Monolayer Graphene. Phys. Rev. Lett. 102, (2009) 235502.

[34] Z. Liu et al. Interlayer shear strength of single crystalline graphite. Acta Mech. Sin. 28, (2012) 978-982. 